\title{
Decentralized Baseband Processing with Gaussian Message Passing Detection for Uplink Massive MU-MIMO Systems
}

\author{
Zhenyu Zhang, Yuanyuan Dong, Keping Long, Senior Member, IEEE, Xiyuan Wang, Member, IEEE, and \\ Xiaoming Dai, Member, IEEE
}

\begin{abstract}
Decentralized baseband processing (DBP) architecture, which partitions the base station antennas into multiple antenna clusters, has been recently proposed to alleviate the excessively high interconnect bandwidth, chip input/output data rates, and detection complexity for massive multi-user multipleinput multiple-output (MU-MIMO) systems. In this paper, we develop a novel decentralized Gaussian message passing (GMP) detection for the DBP architecture. By projecting the discrete probability distribution into a complex Gaussian function, the local means and variances iteratively calculated in each antenna cluster are fused to generate the global symbol beliefs based on the proposed message fusion rule in the centralized processing unit. We present the framework and analysis of the convergence of the decentralized GMP detection based on state evolution under the assumptions of large-system limit and Gaussian sources. Analytical results corroborated by simulations demonstrate that nonuniform antenna cluster partition scheme exhibits higher convergence rate than the uniform counterpart. Simulation results illustrate that the proposed decentralized GMP detection outperforms the recently proposed decentralized algorithms.
\end{abstract}

Index Terms-Massive multi-user multiple-input multipleoutput (MU-MIMO), decentralized baseband processing (DBP), Gaussian message passing (GMP), message fusion, state evolution.

\section{INTRODUCTION}

$\mathbf{M}$ ASSIVE multi-user multiple-input multiple-output (MU-MIMO) systems, in which hundreds of antennas are equipped at the base station (BS), have been extensively investigated owing to the large gains in spectral efficiency, capacity, and reliability over traditional small-scale MIMO systems [1]. However, one of the most critical implementation challenges is the excessively high amount of raw baseband data that must be transferred between the BS radio frequency (RF) units and the baseband processing unit [2], [3]. For instance, the raw baseband data rates exceed $200 \mathrm{Gbit} / \mathrm{s}$ for a massive MU-MIMO BS operating at $40 \mathrm{MHz}$ bandwidth with 128 BS antennas and 10-bit analog-to-digital converters [3], [4]. Such high data rates exceed the bandwidth of existing interconnect technologies and approach the limit of existing

This work was supported by the National Natural Science Foundation of China under Grant 61871029. (Corresponding author: Xiaoming Dai.)

Z. Zhang, Y. Dong, K. Long, and X. Dai are with the School of Computer and Communication Engineering, University of Science and Technology Beijing, Beijing 100083, China (e-mail: zzydq1@163.com; daixiaoming@ustb.edu.cn).

$\mathrm{X}$. Wang is with Beijing Information Science and Technology University, Beijing 100101, China. chip input/output interfaces [5], [6]. Furthermore, classical detection algorithms typically rely on centralized baseband processing. This centralized framework requires full channel state information (CSI) and full received signal, which brings excessively high computational complexity and power consumption for massive MU-MIMO systems [3].

To mitigate this challenge, decentralized baseband processing (DBP) [2]-[6] architecture has been recently proposed for massive MU-MIMO systems. In this decentralized architecture, the BS antennas are partitioned into multiple antenna clusters with independent RF circuitry and computing hardware. Each antenna cluster performs decentralized channel estimation (CHEST) and signal detection, i.e., only the local CSI and received signal are required in each antenna cluster. A centralized processing unit is followed to generate the global estimated symbols based on a given fusion rule for decoding. Reference [2] detailed the decentralized maximum ratio combining (MRC) and minimum mean square error (MMSE) detections, and proposed an optimal fusion rule utilizing the weighted average of local estimates. The MMSE algorithm involves complicated matrix inversion whose computational complexity is cubic to the user number, which is unfavorable in practical implementation. Matrix inversion-less decentralized conjugate gradient (CG) [3], [4], alternating direction method of multipliers (ADMM) [3], and coordinate descent (CD) [5] signal detections have been proposed to reduce the complexity of MMSE detection. However, the bit error rate (BER) performance of these detections only approaches the MMSE method. The authors in [2], [6] proposed a nonlinear detection scheme that builds upon the large-MIMO approximate message passing (LAMA) algorithm, which achieves a slight performance gain over the decentralized MMSE. Note that all these DBPbased detections consider the uniform antenna cluster partition, in which the BS antennas are partitioned equally.

In this paper, we propose an efficient DBP-based detection scheme under the framework of the Gaussian message passing (GMP) algorithm [7]-[9] for uplink massive MUMIMO systems. The GMP algorithm, which is operated on a fully-connected loopy factor graph [10], has been extensively studied for signal detection in massive MU-MIMO systems. In the proposed decentralized GMP detection, each antenna cluster executes independent CHEST and GMP detection in parallel and propagates the local messages to the centralized processing unit. A novel fusion rule is proposed based on the message passing rule to form the global symbol beliefs 
and estimated symbols, rather than using the weighted average scheme proposed in [2], [5]. To prove the convergence of the proposed GMP algorithm, the state evolution is adopted to track the variance variation under the assumptions of largesystem limit and Gaussian sources. Furthermore, we analyze the antenna cluster partition scheme and demonstrate that the nonuniform partition results in a smaller symbol belief variance and higher convergence compared with the uniform partition when fixing the antenna cluster number. Numerical results illustrate that the nonuniform antenna cluster partition scheme achieves performance gain over the uniform counterpart. In addition, the proposed decentralized GMP detection outperforms the recently proposed decentralized algorithms and exhibits linear computational complexity.

The remainder of this paper is organized as follows. Section II describes the system model of uplink massive MU-MIMO with DBP architecture. In Section III, we present the proposed decentralized GMP detection. Simulation results are illustrated in Section IV. Finally, conclusions are drawn in Section V.

Notation: Boldface uppercase letter $\mathbf{X}$ and lowercase letter $\mathbf{x}$ denote matrices and column vectors, respectively. $(.)^{T},(.)^{H}$, $(.)^{-1}$, and $(.)^{*}$ denote the transpose, conjugate transpose, matrix inversion, and complex conjugate, respectively. $\mathbb{N}$ is the set of positive integers. $\mathbf{I}_{N}$ represents the identity matrix with dimension $N . x_{k}$ represents the $k$ th element of $\mathbf{x}$. The $n$th row and $k$ th column element of $\mathbf{X}$ is denoted by $x_{n, k} . \mathbb{E}[$.$] represents the expectation operator. The complex$ Gaussian probability distribution function (PDF) is denoted by $\mathcal{C N}\left(x ; \mu, \sigma^{2}\right)=\left(\pi \sigma^{2}\right)^{-1} \exp \left(-|x-\mu|^{2} / \sigma^{2}\right)$ with mean $\mu$ and variance $\sigma^{2}$.

\section{SySTEM MOdeL}

\section{A. Uplink Massive MU-MIMO Systems}

Consider an uplink massive MU-MIMO system with $K$ single-antenna users simultaneously transmitting to a BS with $N(N \gg K)$ antennas. The complex baseband input-output relation of the uplink massive MU-MIMO channel is given by

$$
\mathbf{y}=\mathbf{H x}+\mathbf{n},
$$

where $\mathbf{y} \in \mathbb{C}^{N \times 1}$ denotes the received signal vector, $\mathbf{H} \in$ $\mathbb{C}^{N \times K}$ is the Rayleigh fading channel matrix whose elements are assumed to be i.i.d. circularly symmetric complex Gaussian distributed with zero mean and unit variance, $\mathbf{x} \in \mathcal{O}^{K \times 1}$ represents the transmitted signal vector whose entries are chosen independently from a power-normalized constellation $\mathcal{O}$ with $|\mathcal{O}|=M$, and $\mathbf{n} \in \mathbb{C}^{N \times 1}$ models the additive white Gaussian noise vector with zero mean and covariance matrix $\sigma_{n}^{2} \mathbf{I}_{N}$. The receiver is assumed to obtain perfect CSI.

\section{B. Decentralized Baseband Processing}

In the DBP architecture, the $N$ BS antennas are partitioned into $C$ independent antenna clusters where $C \in \mathbb{N}$. The $c$ th antenna cluster is equipped with $N_{c}=\omega_{c} N$ antennas where $\omega_{c} \in[0,1], \sum_{c=1}^{C} \omega_{c}=1$, and $N_{c} \in \mathbb{N}$. Each antenna cluster contains local computing hardware, which executes local RF processing, CHEST, and detection in a decentralized

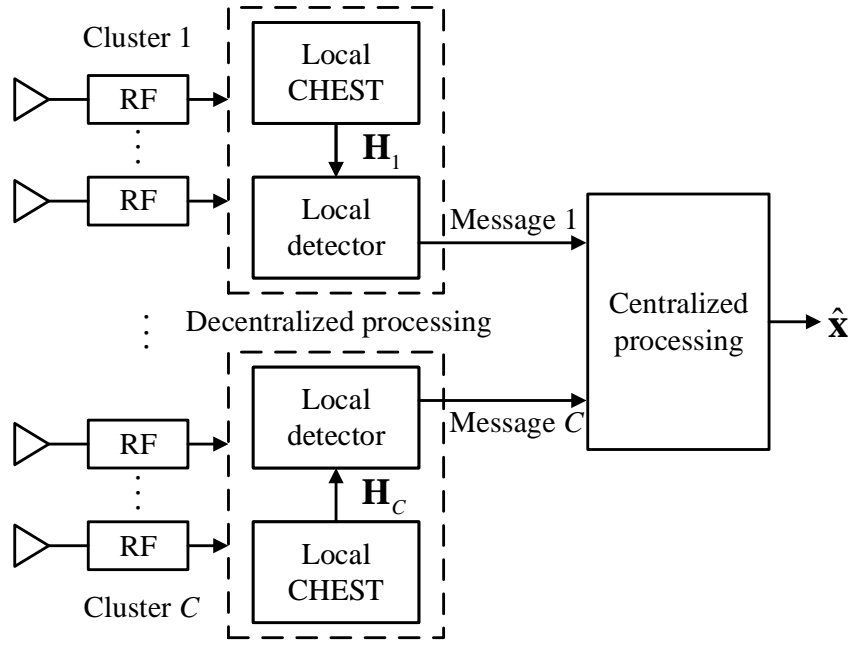

Fig. 1. Uplink massive MU-MIMO system with DBP architecture.

fashion. A fusion node fuses the local information passed from each detector and generates the global estimated symbols. By partitioning the received signal vector as $\mathbf{y}=\left[\mathbf{y}_{1}^{T}, \cdots, \mathbf{y}_{C}^{T}\right]^{T}$, the local received signal associated with the $c$ th antenna cluster is modeled as [2]

$$
\mathbf{y}_{c}=\mathbf{H}_{c} \mathbf{x}+\mathbf{n}_{c}
$$

where the channel matrix is partitioned row-wise into blocks as $\mathbf{H}=\left[\mathbf{H}_{1}^{T}, \cdots, \mathbf{H}_{C}^{T}\right]^{T}$, and the noise vector is partitioned as $\mathbf{n}=\left[\mathbf{n}_{1}^{T}, \cdots, \mathbf{n}_{C}^{T}\right]^{T}$. The massive MU-MIMO with DBP architecture is equivalent to the conventional massive MUMIMO when $C=1$. The block diagram of uplink massive MU-MIMO systems with DBP is depicted in Fig. 1.

\section{Decentralized Gaussian Message Passing Detection FOR MASSIVE MU-MIMO}

\section{A. Decentralized Gaussian Message Passing Detection}

The decentralized GMP detection is operated on a pairwise factor graph [10] consisting of the prior nodes (PNs), variable nodes (VNs), sum nodes (SNs), and fusion nodes (FNs), which denote the mapping constraints, users, likelihood functions, and message fusions, respectively. An example of a factor graph for decentralized GMP detection is shown in Fig. 2 where $N \times K=6 \times 3$ and $C=2$ with uniform partition. Let $\mu_{x_{k} \rightarrow f_{c, n}}^{t}\left(x_{k}\right)$ and $\mu_{f_{c, n} \rightarrow x_{k}}^{t}\left(x_{k}\right)$ denote the messages sent from the $k$ th $(k=1, \cdots, K) \mathrm{VN}$ to the $n$th $\left(n=1, \cdots, N_{c}\right)$ $\mathrm{SN}$ in the $c$ th $(c=1, \cdots, C)$ antenna cluster at the $t$ th $(t=$ $1, \cdots, T)$ iteration and in the opposite direction, respectively, where $T$ is the maximum number of iterations. Based on the sum-product algorithm, the message updating rules are given by [7], [10]

$$
\begin{gathered}
\mu_{x_{k} \rightarrow f_{c, n}}^{t}\left(x_{k}\right)=\mu_{\phi_{k} \rightarrow x_{k}}\left(x_{k}\right) \prod_{n^{\prime} \neq n} \mu_{f_{c, n^{\prime}} \rightarrow x_{k}}^{t-1}\left(x_{k}\right), \\
\mu_{f_{c, n} \rightarrow x_{k}}^{t}\left(x_{k}\right)=\sum_{\mathbf{x} \backslash x_{k}} f_{c, n}\left(y_{c, n} \mid \mathbf{x}\right) \prod_{k^{\prime} \neq k} \mu_{x_{k^{\prime}} \rightarrow f_{c, n}}^{t}\left(x_{k^{\prime}}\right),
\end{gathered}
$$




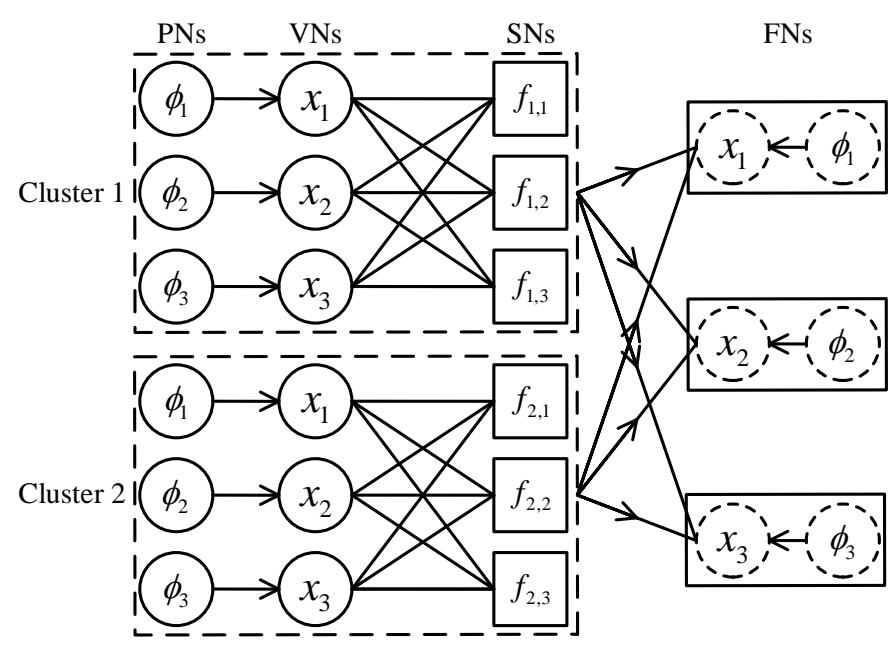

Fig. 2. Factor graph of decentralized GMP detection for $N \times K=6 \times 3$ and $C=2$ with uniform partition.

where $\mathbf{x} \backslash x_{k}$ denotes all the enumerations of $\mathbf{x}$ except for $x_{k}$. The a priori probability is given by $\mu_{\phi_{k} \rightarrow x_{k}}^{t}\left(x_{k}\right)=1 / M$ and the likelihood function is expressed as

$$
f_{c, n}\left(y_{c, n} \mid \mathbf{x}\right)=\frac{1}{\pi \sigma_{n}^{2}} \exp \left(-\frac{1}{\sigma_{n}^{2}}\left|y_{c, n}-\sum_{k} h_{c, n, k} x_{k}\right|^{2}\right),
$$

where $y_{c, n}$ denotes the $n$th element of $\mathbf{y}_{c}$, and $h_{c, n, k}$ denotes the complex channel coefficient from the $k$ th user to the $n$th BS antenna in the $c$ th antenna cluster. We next concentrate on the decentralized GMP detection in the $c$ th antenna cluster.

At the VNs, the summation in (4) contains a global search over the joint space of constellation $\mathcal{O}$ of all users except for the $k$ th user, which results in exponential computational complexity. To alleviate the computational complexity, $x_{k}$ is considered as a continuous random variable and the message $\mu_{f_{c, n} \rightarrow x_{k}}^{t}\left(x_{k}\right)$ is approximated as a complex Gaussian PDF $\mathcal{C N}\left(h_{c, n, k} x_{k} ; m_{f_{c, n} \rightarrow x_{k}}^{t}, v_{f_{c, n} \rightarrow x_{k}}^{t}\right)$. According to the product rule of complex Gaussian PDFs [11], the message passing in (3) is thus computed as

$$
\mu_{x_{k} \rightarrow f_{c, n}}^{t}\left(x_{k}\right) \approx \mu_{\phi_{k} \rightarrow x_{k}}\left(x_{k}\right) \mathcal{C N}\left(x_{k} ; z_{x_{k} \rightarrow f_{c, n}}^{t-1}, \gamma_{x_{k} \rightarrow f_{c, n}}^{t-1}\right) .
$$

The variance and mean are calculated as

$$
\begin{gathered}
\frac{1}{\gamma_{x_{k} \rightarrow f_{c, n}}^{t-1}}=\sum_{n^{\prime} \neq n} \frac{\left|h_{c, n^{\prime}, k}\right|^{2}}{v_{f_{c, n^{\prime}} \rightarrow x_{k}}^{t-1}}, \\
\frac{z_{x_{k} \rightarrow f_{c, n}}^{t-1}}{\gamma_{x_{k} \rightarrow f_{c, n}}^{t-1}}=\sum_{n^{\prime} \neq n} \frac{h_{c, n^{\prime}, k}^{*} m_{f_{c, n^{\prime}} \rightarrow x_{k}}^{t-1}}{v_{f_{c, n^{\prime}} \rightarrow x_{k}}^{t-1}},
\end{gathered}
$$

where $m_{f_{c, n} \rightarrow x_{k}}^{0}=0$ and $v_{f_{c, n} \rightarrow x_{k}}^{0} \rightarrow+\infty$ are initialized. The message $\mu_{x_{k} \rightarrow f_{c, n}}^{t}\left(x_{k}\right)$ is also approximated as a complex Gaussian PDF by minimizing the KL divergence $D_{K L}\left(\mu_{x_{k} \rightarrow f_{c, n}}^{t}\left(x_{k}\right) \| \mathcal{C N}\left(x_{k} ; m_{x_{k} \rightarrow f_{c, n}}^{t}, v_{x_{k} \rightarrow f_{c, n}}^{t}\right)\right)$ for arbitrary prior probability. The mean and variance propagated from VN to $\mathrm{SN}$ are obtained via moment matching

$$
m_{x_{k} \rightarrow f_{c, n}}^{t}=\sum_{\alpha \in \mathcal{O}} \alpha \mu_{x_{k} \rightarrow f_{c, n}}^{t}\left(x_{k}=\alpha\right),
$$

$$
v_{x_{k} \rightarrow f_{c, n}}^{t}=\sum_{\alpha \in \mathcal{O}}|\alpha|^{2} \mu_{x_{k} \rightarrow f_{c, n}}^{t}\left(x_{k}=\alpha\right)-\left|m_{x_{k} \rightarrow f_{c, n}}^{t}\right|^{2} .
$$

At the SNs, the mean and variance propagated from SN to $\mathrm{VN}$ are calculated as [8]

$$
\begin{aligned}
& m_{f_{c, n} \rightarrow x_{k}}^{t}=y_{c, n}-\sum_{k^{\prime} \neq k} h_{c, n, k^{\prime}} m_{x_{k^{\prime}} \rightarrow f_{c, n}}^{t}, \\
& v_{f_{c, n} \rightarrow x_{k}}^{t}=\sigma_{n}^{2}+\sum_{k^{\prime} \neq k}\left|h_{c, n, k^{\prime}}\right|^{2} v_{x_{k^{\prime}} \rightarrow f_{c, n}}^{t} .
\end{aligned}
$$

The means and variances are iteratively propagated between VNs and SNs until $T$ is reached. When the iterative detection process is terminated, the FNs collect the local multiplicative messages $\sum_{n} \mu_{f_{c, n} \rightarrow x_{k}}^{T}\left(x_{k}\right)$, i.e., the local means $m_{f_{c, n} \rightarrow x_{k}}^{T}$, and variances $v_{f_{c, n} \rightarrow x_{k}}^{T}$ from all the antenna clusters to form the global estimation.

At the FNs, the $k$ th $\mathrm{VN}$ gathers information from its input (the $k$ th $\mathrm{PN}$ ) and the SNs of all the antenna clusters to calculate the global symbol belief $\mu_{x_{k}}\left(x_{k}\right)$, i.e., the $a$ posteriori probability. The global symbol belief is given by

$$
\begin{aligned}
\mu_{x_{k}}\left(x_{k}\right) & \propto \mu_{\phi_{k} \rightarrow x_{k}}\left(x_{k}\right) \prod_{c} \prod_{n} \mu_{f_{c, n} \rightarrow x_{k}}^{T}\left(x_{k}\right) \\
& \propto \mu_{\phi_{k} \rightarrow x_{k}}\left(x_{k}\right) \mathcal{C N}\left(x_{k} ; z_{k}, \gamma_{k}\right),
\end{aligned}
$$

where the variance and mean are computed as

$$
\begin{gathered}
\frac{1}{\gamma_{k}}=\sum_{c} \sum_{n} \frac{\left|h_{c, n, k}\right|^{2}}{v_{f_{c, n} \rightarrow x_{k}}^{T}}, \\
\frac{z_{k}}{\gamma_{k}}=\sum_{c} \sum_{n} \frac{h_{c, n, k}^{*} m_{f_{c, n} \rightarrow x_{k}}^{T}}{v_{f_{c, n} \rightarrow x_{k}}^{T}} .
\end{gathered}
$$

The global symbol belief is then normalized as

$$
\mu_{x_{k}}\left(x_{k}=\alpha\right)=\frac{\mu_{\phi_{k} \rightarrow x_{k}}\left(x_{k}=\alpha\right) \mathcal{C N}\left(x_{k}=\alpha ; z_{k}, \gamma_{k}\right)}{\sum_{\alpha^{\prime} \in \mathcal{O}} \mu_{\phi_{k} \rightarrow x_{k}}\left(x_{k}=\alpha^{\prime}\right) \mathcal{C N}\left(x_{k}=\alpha^{\prime} ; z_{k}, \gamma_{k}\right)},
$$

and the global estimated soft symbol is calculated as

$$
\hat{x}_{k}=\sum_{\alpha \in \mathcal{O}} \alpha \mu_{x_{k}}\left(x_{k}=\alpha\right) \text {. }
$$

The proposed decentralized GMP detection for uplink massive MU-MIMO systems is summarized in Algorithm 1.

\section{B. State Evolution Analysis}

To analyze the state evolution of the proposed decentralized GMP detection, we concentrate on the large-system limit (i.e., $N \rightarrow \infty$, fixing the system ratio $K / N$, and fixing $C$ ) and Gaussian sources (i.e., $\mathbf{x} \sim \mathcal{C N}\left(0, \sigma_{x}^{2} \mathbf{I}_{K}\right)$ ). With the symmetry of all the variances [8], [9] in the $c$ th antenna cluster, we assume $\lim _{t \rightarrow+\infty} v_{x_{k} \rightarrow f_{c, n}}^{t}=v_{x \rightarrow f}^{c}, \lim _{t \rightarrow+\infty} v_{f_{c, n} \rightarrow x_{k}}^{t}=v_{f \rightarrow x}^{c}$, and $\mathbb{E}\left[\left|h_{c, n, k}\right|^{2}\right] \approx 1$ for $\forall k \in\{1, \cdots, K\}$ and $\forall n \in\left\{1, \cdots, N_{c}\right\}$ in the large-system limit. When Gaussian sources are assumed, the variance $v_{x_{k} \rightarrow f_{c, n}}^{t}$ of $\mu_{x_{k} \rightarrow f_{c, n}}^{t}\left(x_{k}\right)$ (6) is computed as

$$
\frac{1}{v_{x_{k} \rightarrow f_{c, n}}^{t}}=\frac{1}{\sigma_{x}^{2}}+\sum_{n^{\prime} \neq n} \frac{\left|h_{c, n^{\prime}, k}\right|^{2}}{v_{f_{c, n^{\prime}} \rightarrow x_{k}}^{t}} \text {. }
$$




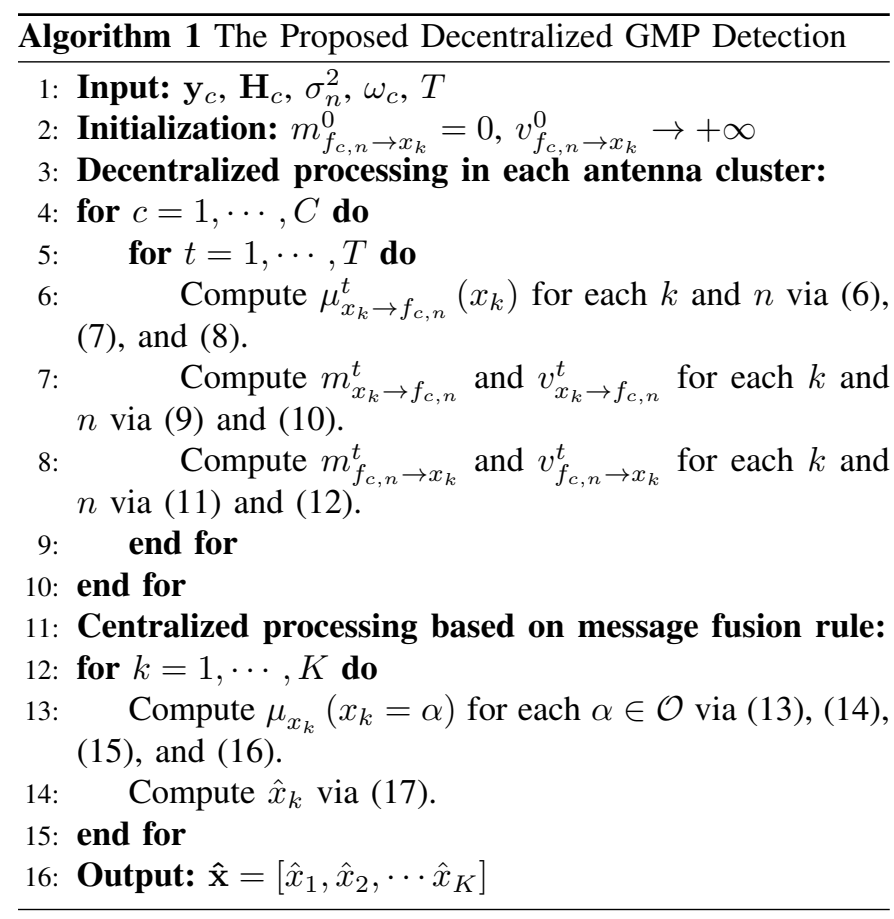

When $t \rightarrow+\infty$, taking the expectations of (12) and (18) results in [8]

$$
\begin{gathered}
v_{f \rightarrow x}^{c}=\sigma_{n}^{2}+K v_{x \rightarrow f}^{c}, \\
\frac{1}{v_{x \rightarrow f}^{c}}=\frac{1}{\sigma_{x}^{2}}+\frac{\omega_{c} N}{v_{f \rightarrow x}^{c}} .
\end{gathered}
$$

Combining (19) and (20), we have the following equation

$$
v_{f \rightarrow x}^{c}{ }^{2}+\left(\omega_{c} N \sigma_{x}^{2}-K \sigma_{x}^{2}-\sigma_{n}^{2}\right) v_{f \rightarrow x}^{c}-\omega_{c} N \sigma_{x}^{2} \sigma_{n}^{2}=0 .
$$

The fix point is computed as the positive solution

$v_{f \rightarrow x}^{c}=\frac{K \sigma_{x}^{2}-\omega_{c} N \sigma_{x}^{2}+\sigma_{n}^{2}+\sqrt{\left(\omega_{c} N \sigma_{x}^{2}-K \sigma_{x}^{2}+\sigma_{n}^{2}\right)^{2}+4 K \sigma_{x}^{2} \sigma_{n}^{2}}}{2}$.

When considering Gaussian sources, the global symbol belief is also a Gaussian function whose variance $v$ is calculated as

$$
\begin{aligned}
& \frac{1}{v}=\frac{1}{\sigma_{x}^{2}}+\sum_{c} \frac{\omega_{c} N}{v_{f \rightarrow x}^{c}}=\frac{1}{\sigma_{x}^{2}}+ \\
& \sum_{c} \frac{2 \omega_{c} N}{K \sigma_{x}^{2}-\omega_{c} N \sigma_{x}^{2}+\sigma_{n}^{2}+\sqrt{\left(\omega_{c} N \sigma_{x}^{2}-K \sigma_{x}^{2}+\sigma_{n}^{2}\right)^{2}+4 K \sigma_{x}^{2} \sigma_{n}^{2}}} .
\end{aligned}
$$

\section{Partition Scheme of the Antenna Clusters}

The variance of the symbol belief affects the convergence rate of the proposed decentralized GMP detection. A smaller variance results in a larger probability for the most probable constellation symbol, which accelerates the convergence rate of the symbol belief. We define the function $f(x)$ as

$$
f(x)=\frac{2 x N}{K \sigma_{x}^{2}-x N \sigma_{x}^{2}+\sigma_{n}^{2}+\sqrt{\left(x N \sigma_{x}^{2}-K \sigma_{x}^{2}+\sigma_{n}^{2}\right)^{2}+4 K \sigma_{x}^{2} \sigma_{n}^{2}}},
$$

TABLE I

COMPUTATIONAL COMPLEXITY.

\begin{tabular}{c||c}
\hline \hline Algorithms & Number of complex multiplications \\
\hline GMP & $(6 M T N+8 T N+N+5 M) K$ \\
\hline LAMA & $(N+C T) K^{2}+(N+5 C M T+2 C T+3 C) K$ \\
\hline MMSE & $\frac{10}{3} C K^{3}+\left(N+\frac{7}{2} C\right) K^{2}+\left(N+\frac{13}{6} C\right) K$ \\
\hline MRC & $C K^{3}+(N+3 C) K^{2}+(N+5 C) K$ \\
\hline \hline
\end{tabular}

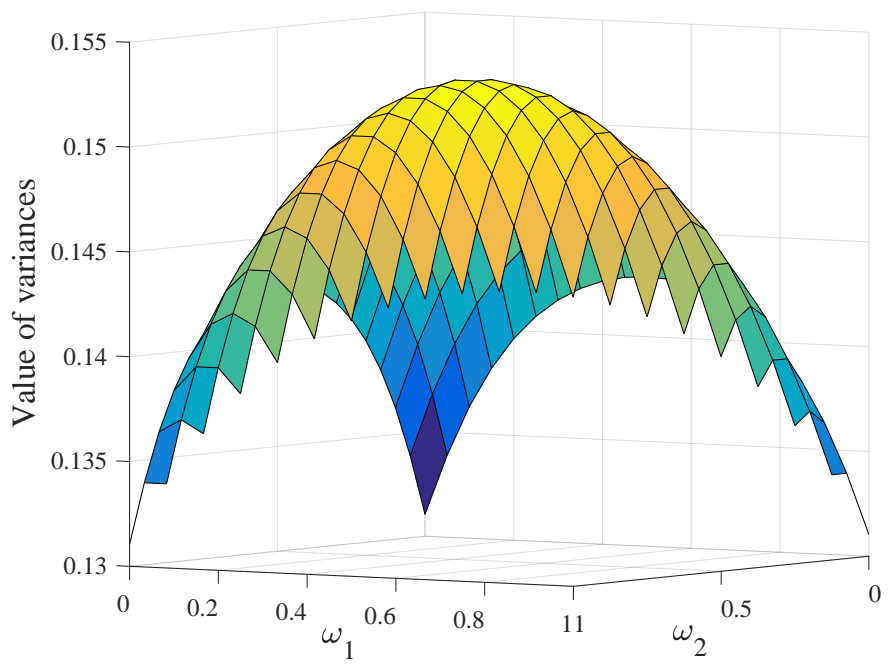

Fig. 3. Variance variation of symbol beliefs versus $\omega$ at $\mathrm{SNR}=0 \mathrm{~dB}$ where $K=16$ and $C=3$ with uniform partition.

where $x \in[0,1]$. Note that $f(x)$ is convex in $x$ as the second order derivative $f^{\prime \prime}(x)>0$. According to the Jensen inequality, we obtain that

$$
\frac{1}{C} \sum_{c=1}^{C} f\left(\omega_{c}\right) \geq f\left(\frac{1}{C} \sum_{c=1}^{C} \omega_{c}\right)
$$

where equality holds if $\omega_{1}=\omega_{2}=\cdots=\omega_{C}$. This indicates that the uniform partition results in the slowest convergence rate of the symbol belief. From (25) we obtain $\sum_{c=1}^{C} f\left(\omega_{c}\right) \geq$ $C f(1 / C)$. We define the function $g(x)=f(x) / x$. Note that $g(x)$ is monotonically increased with the increase of $x$ as the first order derivative $g^{\prime}(x)>0$. This indicates that a smaller number of antenna clusters results in a faster convergence rate of the symbol belief when using the uniform partition.

\section{Computational Complexity Analysis}

The computational complexity is analyzed in terms of the required number of complex multiplications. The complexity of the decentralized processing is $N K+8 T N K+6 M T N K$ while the centralized processing requires $5 M K$ complex multiplications. The computational complexity of the proposed decentralized GMP detection and the recently proposed decentralized algorithms is summarized in Table I.

\section{Simulation Results}

In this section, we evaluate the BER performance and computational complexity of the proposed decentralized GMP detection. The recently proposed decentralized LAMA, MMSE, 


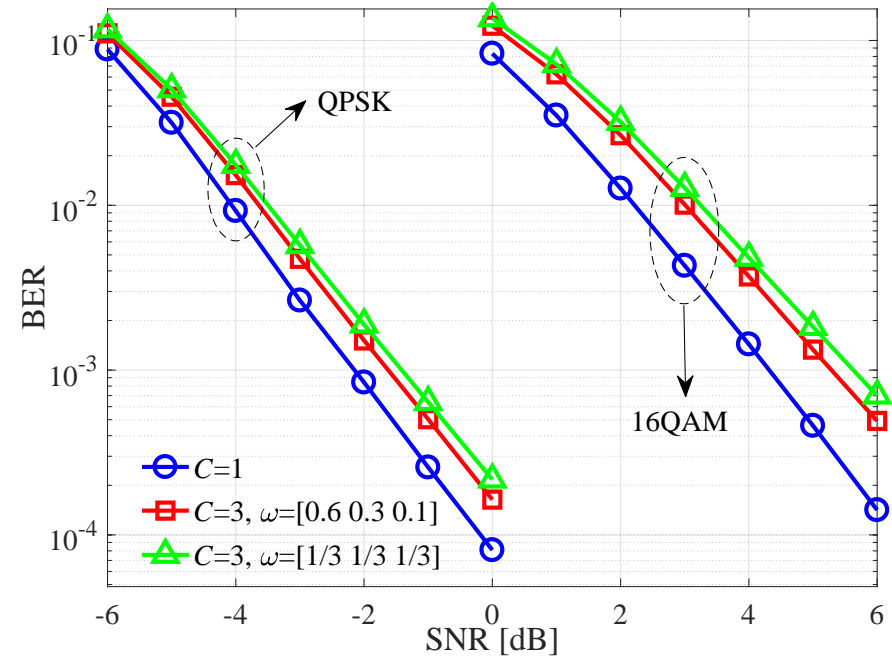

Fig. 4. BER performance of the decentralized GMP detection for different antenna cluster partition schemes where $K=16$.

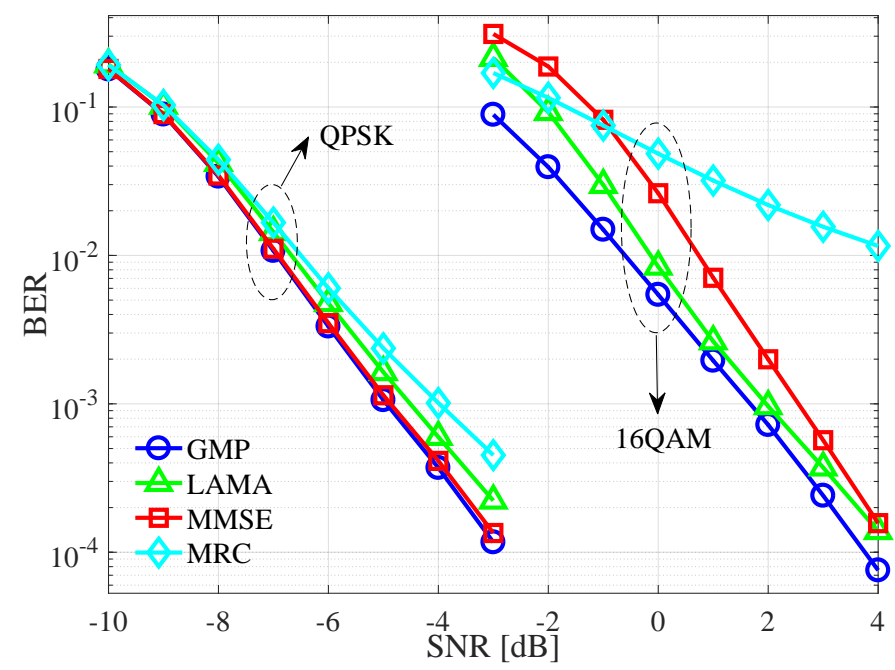

Fig. 5. BER performance of detection algorithms where $K=8$ and $C=3$ with uniform partition.

and MRC algorithms are compared as benchmarks. The BS is equipped with $N=120$ antennas. The convolutional code of rate $1 / 2$ is adopted. The maximum number of iterations for the decentralized GMP and LAMA methods is set as $T=5$.

Fig. 3 shows the variance variation of symbol beliefs versus $\omega$ at $\mathrm{SNR}=0 \mathrm{~dB}$, where $K=16$ and $C=3$ with uniform partition. This figure further illustrates that the uniform partition leads to the largest variances. Fig. 4 evaluates the BER performance of the proposed decentralized GMP detection with different antenna cluster partition schemes for $C=3$. The decentralized processing with nonuniform antenna cluster partition outperforms the uniform counterpart and approaches the centralized processing with acceptable performance loss.

To prove the efficiency of the proposed decentralized GMP detection, the BER performance comparison with the state-ofthe-art decentralized algorithms is evaluated in equally-sized antenna clusters which are desirable in practice as the uniform partition minimizes the interconnect or chip input/output bandwidth as well as the computational complexity per computing

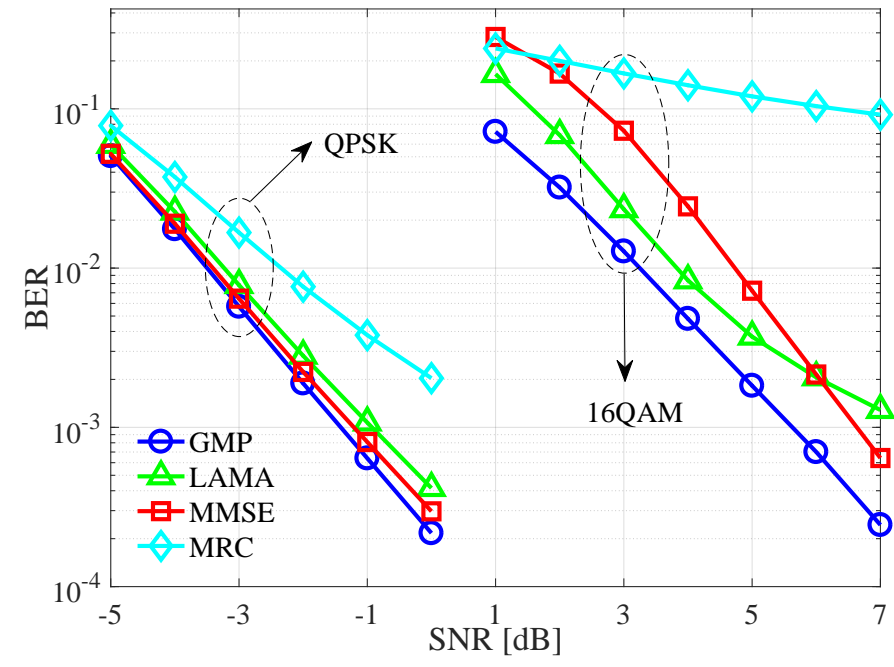

Fig. 6. BER performance of detection algorithms where $K=16$ and $C=3$ with uniform partition.

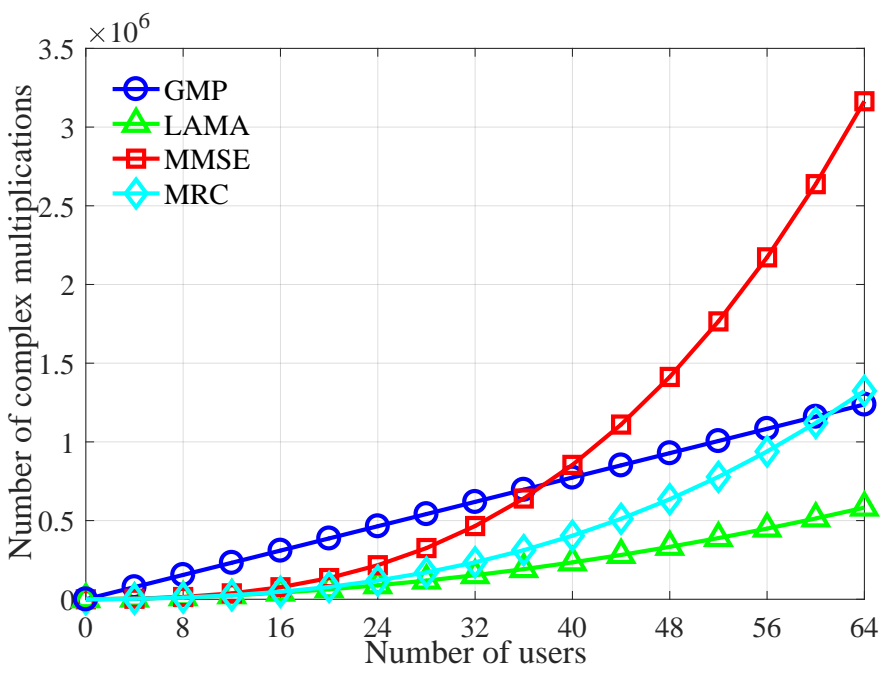

Fig. 7. Computational complexity comparison where $C=3$ and QPSK.

fabric [2]. Fig. 5 and Fig. 6 illustrate that the proposed decentralized GMP detection outperforms the other methods, especially for high modulation order and large number of user. For example, the decentralized GMP achieves gains of nearly $1.1 \mathrm{~dB}$ and $2.1 \mathrm{~dB}$ over the decentralized MMSE and LAMA at BER of $10^{-3}$, respectively, for $K=16$ and 16QAM.

Fig. 7 presents the complexity comparison which shows that the proposed decentralized GMP detection behaves linear complexity with the increase of user number. This indicates that the implementation of the proposed algorithm exhibits hardwarefriendly complexity in the case of massive connection.

\section{CONClusion}

This paper proposed a novel decentralized GMP detection for uplink massive MU-MIMO systems with DBP. The centralized processing executes the message fusion by gathering the local messages propagated from each antenna cluster in parallel. The convergence of the proposed algorithm is characterized by state evolution under the assumptions of large-system limit and Gaussian sources. We demonstrated 
that the nonuniform partition outperforms the uniform partition for a fixed antenna cluster number. Simulation results showed that the proposed decentralized GMP detection outperforms the recently proposed methods and exhibits linear complexity.

\section{REFERENCES}

[1] T. L. Marzetta, "Noncooperative Cellular Wireless with Unlimited Numbers of Base Station Antennas," IEEE Trans. Wireless Commun. vol. 9, no. 11, pp. 3590-3600, November 2010.

[2] C. Jeon, K. Li, J. R. Cavallaro, and C. Studer, "Decentralized Equalization With Feedforward Architectures for Massive MU-MIMO," IEEE Trans. Signal Process., vol. 67, no. 17, pp. 4418-4432, 2019.

[3] K. Li, R. R. Sharan, Y. Chen, T. Goldstein, J. R. Cavallaro, and C. Studer "Decentralized Baseband Processing for Massive MU-MIMO Systems," IEEE J. Emerg. Sel. Topics Circuits Syst., vol. 7, no. 4, pp. 491-507, Dec. 2017.

[4] K. Li, Y. Chen, R. Sharan, T. Goldstein, J. R. Cavallaro, and C. Studer, "Decentralized data detection for massive MU-MIMO on a Xeon Phi cluster," in Proc. 50th Asilomar Conf. Signals, Syst. Comput., Pacific Grove, CA, 2016, pp. 468-472.

[5] K. Li, O. Castaneda, C. Jeon, J. R. Cavallaro, and C. Studer, "Decentralized coordinate-descent data detection and precoding for massive MU-MIMO," in Proc. IEEE Int. Symp. Circuits Syst., Sapporo, Japan, 2019 , pp. $1-5$

[6] C. Jeon, K. Li, J. R. Cavallaro, and C. Studer, "On the achievable rates of decentralized equalization in massive MU-MIMO systems," in Proc. IEEE Int. Symp. Inf. Theory, Aachen, 2017, pp. 1102-1106.

[7] S. Wu, L. Kuang, Z. Ni, J. Lu, D. Huang, and Q. Guo, "Low-Complexity Iterative Detection for Large-Scale Multiuser MIMO-OFDM Systems Using Approximate Message Passing," IEEE J. Sel. Topics Signal Process., vol. 8, no. 5, pp. 902-915, Oct. 2014.

[8] L. Liu, C. Yuen, Y. L. Guan, Y. Li, and Y. Su, "Convergence Analysis and Assurance for Gaussian Message Passing Iterative Detector in Massive MU-MIMO Systems," IEEE Trans. Wireless Commun., vol. 15, no. 9, pp. 6487-6501, 2016.

[9] L. Liu, C. Yuen, Y. L. Guan, Y. Li, and C. Huang, "Gaussian Message Passing for Overloaded Massive MIMO-NOMA," IEEE Trans. Wireless Commun., vol. 18, no. 1, pp. 210-226, 2019.

[10] F. R. Kschischang, B. J. Frey, and H.-A. Loeliger, "Factor graphs and the sum-product algorithm," IEEE Trans. Inf. Theory, vol. 47, no. 2, pp. 498-519, Feb. 2001.

[11] P. Bromiley, "Products and convolutions of Gaussian probability density functions," Tina-Vision Memo Tech. Rep. 2003-003, 2003. 